\title{
ARE CHARACTERISTICS OF SOFT PALM STEARINS SIMILAR TO SOFT PALM MID FRACTIONS?
}

\author{
SIVARUBY KANAGARATNAM*; NOOR LIDA HABI MAT DIAN*; RAFIDAH ABD HAMID*; \\ NUR HAQIM ISMAIL*; WAN ROSNANI AWG ISA*; NORAZURA AILA MOHD HASSIM*; \\ ZALIHA OMAR* and MISKANDAR MAT SAHRI*
}

\begin{abstract}
The aim of this assessment was to determine the variation between soft palm mid fractions (sPMF) and soft palm stearins (sPOs) which are the co-fractions (solid fractions) obtained from two different fractionation processes which yield palm olein of iodine value (IV) 60. The sPMF and sPOs are not governed by any official standard specifications and are traded based on contractual specifications. Furthermore, the physical and chemical characteristics of sPOs lack documentation as it falls into the general category of palm stearin. Hence, users who are unaware of the differences between these two fractions are misinformed and misguided, leading to dysfunctional application of these fractions in food products. This assessment of sPMF and sPOs evaluated the triacylglycerol composition, fatty acid compositions (FAC), IV, dropping point (DP), solid fat content (SFC) profiles and thermal behaviours in order to establish parameters which can be used as quality qualifiers, which are able to distinctively discriminate both fractions. The triacylglycerol compositions differed significantly between sPMF and sPOs. sPMF contained high amounts of monounsaturated triacylglycerols ranging from $61.83 \%$ to $80.07 \%$ with 1,3-dipalmitoyl-2-oleoyl-glycerol (POP) ranging from $43.53 \%$ to $55.95 \%$. Lesser amount of monounsaturated triacylglycerols were present in sPOs, ranging from $55.56 \%$ to $57.33 \%$ (POP from $38.36 \%$ to $41.81 \%$ ). The trisaturated triacylglycerols ranged from $2.45 \%$ to $5.41 \%$ in sPMF and higher values were detected in sPOs ranging from $10.46 \%$ to $18.52 \%$. SFC profiles of sPMF and sPOs varied significantly. The sPMF exhibited a sharp decrease in the percentage of solids from $20^{\circ} \mathrm{C}$ to $30^{\circ} \mathrm{C}$ which were not exhibited by sPOs. The DP of sPMF ranged between $24.3^{\circ} \mathrm{C}$ to $31.8^{\circ} \mathrm{C}$ and sPOs varied between $40.3^{\circ} \mathrm{C}$ to $50.1^{\circ} \mathrm{C}$. sPMF and sPOs exhibit unique and distinctive crystallisation and melting profiles which can be used to differentiate these two fractions. Fatty acid composition and iodine values exhibited overlapping values, hence, are inadequate indicators to differentiate these fractions. The assessment concludes that triacylglycerol composition, DP, SFC profile and thermal behaviour (crystallisation and melting) are indicative parameters that are able to assist in differentiating the physical and chemical characteristics of sPMF and sPOs.
\end{abstract}

Keywords: soft palm mid fraction, soft palm stearin, 1-3-dipalmitoyl-2-oleoyl-glycerol (POP).

Date received: 4 January 2019; Sent for revision: 6 January 2019; Accepted: 20 May 2019.

\section{INTRODUCTION}

Oil palm has emerged as a major economic crop feeding the world with an overwhelming production

\footnotetext{
* Malaysian Palm Oil Board,

6 Persiaran Institusi, Bandar Baru Bangi,

43000 Kajang, Selangor, Malaysia.

E-mail: sivaruby@mpob.gov.my
}

of 67.92 million tonnes in 2017. A major portion of palm oil amounting to $80 \%$ of production is channelled towards supporting the global demand of the food sector (Kushari et al., 2018). Refined palm oil to a great extent is fractionated to olein (liquid) and stearin (solid) fractions, as these fractions have distinctive roles in the food sector. Palm olein and palm stearin undergo single or multiple 
fractionation steps to fulfil the specific application and functionality requirements of the food sector.

Soft palm mid fraction (sPMF) and soft palm stearin (sPOs) are co-fractions (solid fractions) obtained from the fractionation process to produce palm olein of iodine value (IV) 60. Figure 1 shows the flow charts of the two routes for obtaining palm olein of IV 60, the common routes practiced by the Malaysian fractionation plants. The first route is fractionation of palm olein IV 56, which will yield palm olein IV 60 and sPMF (Danthine et al., 2017; Gijs et al., 2007; Calliauw et al., 2007; Hashem et al., 2018; Kellens et al., 2007). The second route is single fractionation of palm oil (IV 50 to 52) which yields palm olein IV 60 and sPOs. The latter is cost-effective and is preferred by the industry, as this process shortens the production time, hence, improves productivity. Nevertheless, sPMF is a premium grade product, in contrast to sPOs which is traded as the general grade of palm stearin.

The sPMF and sPOs are not governed by any official standard specifications and are traded based on contractual specifications. The concern arises as some of the contractual specifications are not able to discriminated between sPMF and sPOs. Iodine values are generally included in the contractual specifications for trading of sPMF and sPOs. The non-significantly difference iodine values of sPMF and sPOs stands as a challenge to the potential buyers as it may cause confusion. Hence,
sPMF and sPOs are two solid fractions which are sometimes misinterpreted by food formulators, who are unaware of the differences between these two fractions, which in some cases have led to dysfunctional application of these fractions in food products.

Palm mid fractions (PMF) are categorised as specialty fats due to their unique sharp melting characteristic which suits special functional applications in food formulations (Gee, 2007; Sonwai et al., 2014). PMF are widely used as cocoa butter equivalent, coating fats, fat component of margarine and shortening (Kellens et al., 2007; Lumor et al., 2010; Biswas et al., 2017; Jahurul et al., 2014). The fractionation process increases the crucial triacylglycerol which is 1,3-dipalmitoyl-2oleoyl-glycerol (POP) content from approximately $27 \%$ in palm olein to above $40 \%$ in the sPMF. The characteristics of sPMF are dominated by the amount of POP present in the fractions. Nevertheless, the presences of other triacylglycerols have an impact on the overall characteristics of the fractions. The sPMF is further fractionated to obtain solid fraction which is known and traded as hard palm mid fraction, a value-added premium fraction. The key variation between these two fractions is the amount of POP, the amount of POP in hard palm mid fractions are more than $65 \%$.

The sPOs are least documented as these fractions are categorised as general grade of palm stearin.

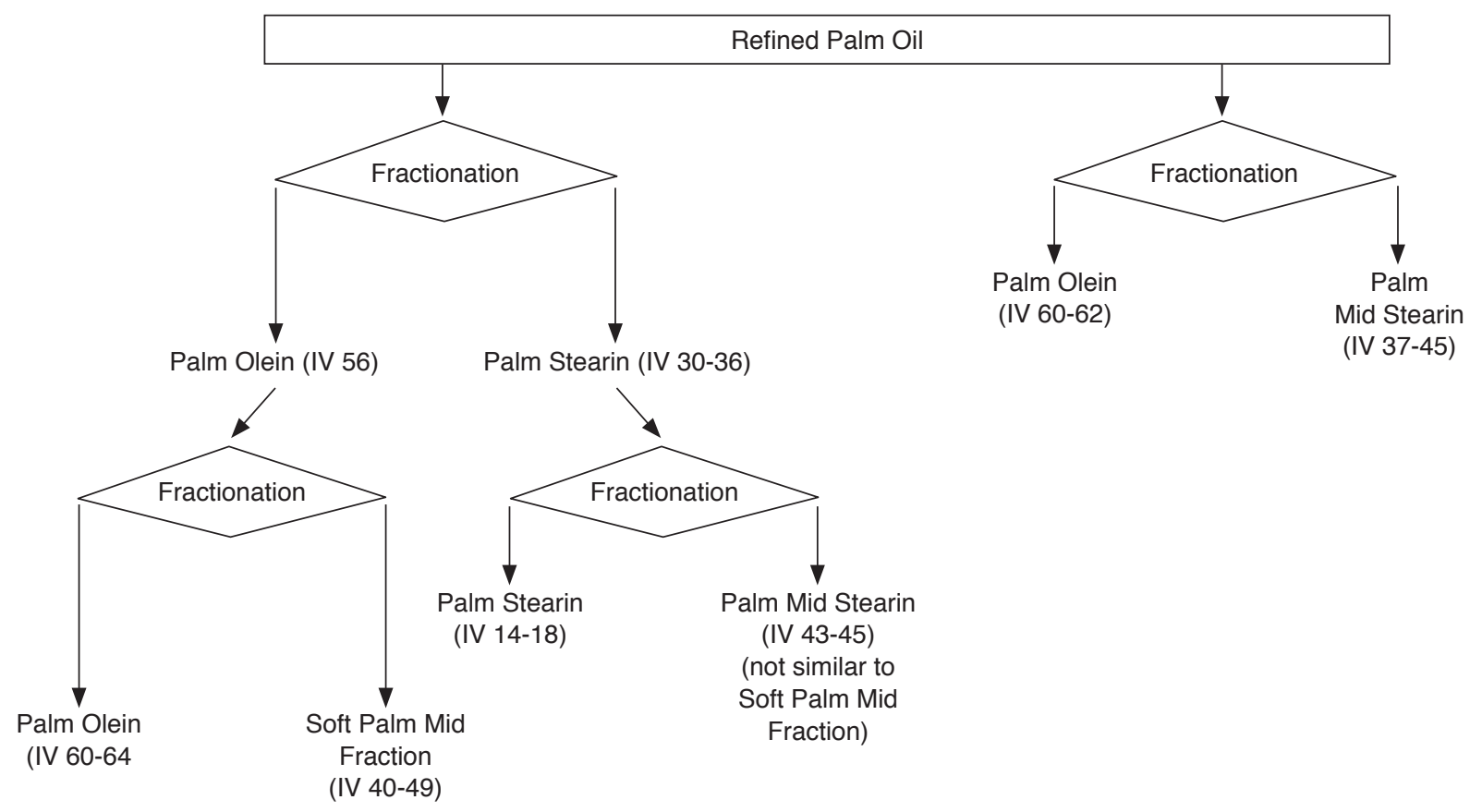

Note: IV - iodine value.

Source: Information from the Malaysian fractionation plants operators: Sime Dardy Jomalina Sdn Bhd, Agri Asia Refinery Sdn Bhd, Sawit Raya Sdn Bhd, SOP Edible Oils Sdn Bhd, Premium Vegetable Oil Sdn Bhd, Mewah Oils Sdn Bhd Malaysia, Ecolex Sdn Bhd and Moi Foods Malaysia Sdn Bhd.

Figure 1. Fractionation process flow of production of soft palm mid fraction (sPME) and soft palm stearin (sPOs). 
Most palm stearin from Malaysia is traded based on the Palm Oil Refiners' Association Malaysia (PORAM) specifications, which specifies palm stearin as fractions with IV of 48 maximum and melting point value of $44^{\circ} \mathrm{C}$ minimum (PORAM Specification., 2013). Palm stearins are very costeffective, natural and non-hydrogenated fats which are commonly and widely used in the production of frying fats, shortening, margarine, vegetables ghee (vanaspati), dairy fat replacers and animal fat replacers (Adhikari et al., 2010; Sivaruby et al., 2018).

The application of fats in products such as in cocoa butter alternatives, margarine, shortenings, replacers of dairy fats and replacers of animal fats to a large extent is influenced by the triacylglycerol compositions (Biswas et al., 2017). Triacylglycerol composition determines the characteristics of the fats with respect to their macroscopic properties such as overall texture which in turn affects the compatibility of the fats into various food systems. It is well documented by other researchers that small variation in triacylglycerol composition gives significant changes to the overall functionality and application of the fats in the final food products. Changes in triacylglycerol composition will cause changes in melting and crystallisation behaviour, which determines the structure, crystal morphology and polymorphic transitions of the crystals, hence, leads to variation in the crystal network (Narine and Marangoni, 1999; Marangoni, 2005). Triacylglycerols are basically structural component of fats, thus, variation in triacylglycerol composition will lead to major changes in the characteristics of the functionality of the fats (Vereecken et al., 2010). Hence, structure to functionality relationship is of great importance in the selection of fats for food formulation.

The aim of this evaluation was to determine and discuss the variations and similarities in the physical and chemical characteristics of sPMF and sPOs. The analyses of interest in determining the physical and chemical characteristics of sPMF and sPOs were triacylglycerol composition, fatty acid composition (FAC), IV, dropping point (DP), solid fat content (SFC) profile and thermal behaviour of the fractions. These analyses were able to indicate and identify the variations and similarities of these fractions. Hence, enables differentiation of these fractions by establishing their unique characteristics.

\section{MATERIALS AND METHODS}

\section{Materials}

The sPMF and sPOs samples were obtained from the Malaysian fractionation plants. The samples obtained were produced via dry fractionation process.

\section{Triacylglycerol Composition Analysis}

Triacylglycerol analysis was determined using the method reported in Ramli et al. (2008). Samples were prepared by dissolving $0.015 \mathrm{~g} \mathrm{litre}^{-1}$ in acetone. Solid impurities were removed by filtering the samples through a $0.2 \mu \mathrm{m}$ nylon membrane filter. High performance liquid chromatography (Agilent 1100 Series, USA) was used to determine the triacylglycerol composition. Two Purospher ${ }^{\circledR}$ STAR RP-18 end-capped column ( $25 \mathrm{~cm}$ length $\times 4 \mathrm{~mm}$ i.d with of $5 \mu \mathrm{m}$ particle size) (Merck KGaA, Darmtadt, Germany) were connected in series. Sample of 10 $\mu \mathrm{l}$ was injected into the column and column was kept in an oven at $30^{\circ} \mathrm{C}$. The mobile phase used was acetone:acetonitrile (75:25\% vol/vol), at a flow rate of $1.0 \mathrm{ml} \mathrm{min} \mathrm{m}^{-1}$ under isocratic conditions. The individual peaks were identified by a refractive index detector and the total run time was $45 \mathrm{~min}$ per sample. The chromatograms were identified by comparing retention times with standards and quantified using the area normalisation method (Ramli et al., 2008).

\section{Fatty Acid Composition}

FAC was determined as fatty acid methyl esters. The samples $(0.05 \mathrm{~g})$ were weighed and dissolved in $1 \mathrm{ml}$ hexane. The mixture was then added with sodium methoxide solution, $0.2 \mathrm{ml}$ of $\mathrm{NaOCH}_{3}(2 \mathrm{M})$, in anhydrous methanol and then mixed for $1 \mathrm{~min}$ with a vortex mixer. After sedimentation of sodium glycerolate, $1 \mu \mathrm{l}$ of clear supernatant was injected into Rtx 2330 fused silica capillary column (60 m $\times 0.25 \mathrm{~mm} \times 0.25 \mathrm{~mm}$ ) (Restex Corporation, USA) and analysed using a Burker Gas Chromatography system Model 430-GC (Burker Daltonics, CA, USA) equipped with a flame ionisation detector (FID) and Galaxie Chromatography Data System. Injection and detection temperatures were set at $240^{\circ} \mathrm{C}$. The oven temperature was set at $190^{\circ} \mathrm{C}$. The column temperature was isothermal at $185^{\circ} \mathrm{C}$. The carrier gas was helium with a flow rate of $1 \mathrm{ml} \mathrm{min}^{-1}$. The peaks were identified by comparing retention times with FAME standards and quantified using peak area normalisation methods.

\section{Iodine Value}

The IV denotes the degree of unsaturation of fatty acids in oils and fats. Determination of IV was carried out according to the MPOB Test Method MPOB p3.2:2004 (Kuntom et al., 2005). The $20 \mathrm{~g}$ of totally melted and homogenised sample and 20 $\mathrm{ml}$ of solvent (1:1 cyclohexane:glacial acetic acid) were weighed into a conical glass flask fitted with a round glass stopper. Followed by $25 \mathrm{ml}$ of Wijs reagent was added, the stopper was placed, the solution swirled by hand and was placed in a dark 
room for $1 \mathrm{hr}$. A blank test was prepared by omitting the test portion. At the end of the reaction time, 20 $\mathrm{ml}$ of potassium iodide and $150 \mathrm{ml}$ of water were added. The solution was then titrated with standard sodium thiosulphate solution. The blank test was similarly treated and titrated. Calculation of the IV was carried out and the IV were expressed as $g$ of $\mathrm{I}_{2} / 100 \mathrm{~g}$ of oil.

$$
\mathrm{IV}=\frac{12.69 \mathrm{C}\left(\mathrm{V}_{1}-\mathrm{V}_{2}\right)}{\mathrm{M}}
$$

$\mathrm{C}$ is concentration of sodium thiosulphate.

$\mathrm{V}_{1}$ is volume, in millilitres, of sodium thiosulphate solution used in blank test.

$\mathrm{V}_{2}$ is volume, in millilitres, of sodium thiosulphate solution used in determination of sample.

$\mathrm{M}$ is the mass, in grams, of sample.

\section{Dropping Point}

The DP indicates the temperature where the frozen samples become liquid and are able to flow. Determination of DP was carried out in accordance to MPOB Test Method f1.2 (Kuntom et al., 2005). A Mettler Toledo DP analysing system with central processor FP90 (AG, Schwerzenvach, Switzerland) was used to determine the DP of the samples. The sample cup (inner diameter $2.8 \mathrm{~mm}$ Mettler No. 18732) was placed in the freezer (below $-5^{\circ} \mathrm{C}$ ) for 10 min. The sample was melted, homogenised and filled into the sample cup. The filled cup was kept in the freezer for $15 \mathrm{~min}$. The DP analyser was set at $10^{\circ} \mathrm{C}$ below the expected DP. The heating rate was set at $1^{\circ} \mathrm{C} \mathrm{min}^{-1}$. The sample was removed from the freezer and immediately placed into the DP measuring cell and the DP values were recorded (Sivaruby et al., 2013; Kuntom et al., 2005).

\section{Solid Fat Content}

SFC denotes the amount of solid present at a specific temperature. Determination of SFC was performed according to the MPOB Test Method p4.8:2004 (Kuntom et al., 2005). The SFC was measured with Bruker Minispec PC 120 pulseNMR. Samples were totally melted at $80^{\circ} \mathrm{C}$ to erase all crystal memory. The totally melted samples were homogenised and filled into tubes $(10 \mathrm{~mm}$ o.d $\mathrm{x}$ $75 \mathrm{~mm}$ length) up to $3 \mathrm{~cm}$ in height. The samples were tempered at $70^{\circ} \mathrm{C}$ in a water bath for $30 \mathrm{~min}$, before chilling at $0^{\circ} \mathrm{C}$ for $90 \mathrm{~min}$. These tubes were conditioned in pre-equilibrated thermostated baths for $30 \mathrm{~min}$ prior to the measurement. The measuring temperatures were $0^{\circ} \mathrm{C}, 5^{\circ} \mathrm{C}, 10^{\circ} \mathrm{C}, 15^{\circ} \mathrm{C}$, $20^{\circ} \mathrm{C}, 25^{\circ} \mathrm{C}, 30^{\circ} \mathrm{C}, 35^{\circ} \mathrm{C}, 40^{\circ} \mathrm{C}, 45^{\circ} \mathrm{C}, 50^{\circ} \mathrm{C}, 55^{\circ} \mathrm{C}$ and $60^{\circ} \mathrm{C}$. The direct method was employed for the measurements.

\section{Thermal Behaviour}

Differential scanning calorimetry (DSC) analyses were carried out using a Perkin Elmer DSC Model DSC-7 with refrigerated cooling system (Perkin Elmer, Norwalk, CT). The instrument was attached to a data processing unit, Perkin Elmer Thermal Data Station. Calibration was carried out with an indium standard. The 4 to $5 \mathrm{mg}$ of a precisely weighed $( \pm 0.1 \mathrm{mg})$ fat sample was placed in the DSC pan and hermetically sealed. The cooling and heating profiles of samples were defined by using two different temperature programs. The samples were subjected to the following temperature profile: $70^{\circ} \mathrm{C}$ for $5 \mathrm{~min}$, cooled at a cooling rate of $10^{\circ} \mathrm{C} \mathrm{min}{ }^{-1}$ to $-40^{\circ} \mathrm{C}$ and the cooling thermogram was recorded. The same samples were held at $-40^{\circ} \mathrm{C}$ for $5 \mathrm{~min}$ then heated from $-40^{\circ} \mathrm{C}$ to $70^{\circ} \mathrm{C}$ at a heating rate of $10^{\circ} \mathrm{C}$ $\mathrm{min}^{-1}$ and the melting thermogram was recorded.

\section{Statistical Analysis}

All measurements were performed in triplicate unless stated and data were reported as mean \pm standard deviations of independent analysis. The significant difference of the samples was analysed using a one-way analysis of variance (AVONA) by Tukey's test at a significance level of 0.02. All statistical analyses were computed using Minitab 16.0 (Minitab Inc, Pennsylvania, USA).

\section{RESULTS AND DISCUSSION}

\section{Triacylglycerol Composition}

The eight sPMF samples collected from the Malaysian fractionation plants were produced via fractionation of palm olein IV 56. sPMF contain high amounts of monounsaturated triacylglycerols ranging from $61.83 \%$ to $80.07 \%$ with the most abundant triacylglycerol being POP ranging from $43.53 \%$ to $55.95 \%$, as shown in Table 1 . The amount of POP reported by Sonwai's team was $51.8 \%$, Biswas's team reported $50.7 \%$ and Calliauw's team reported two values, which were $40.75 \%$ and $48.90 \%$ (Sonwai et al., 2014; Biswas et al., 2017; Calliauw et al., 2007 ). POP is the crucial triacylglycerol in PMF, as it dominantly contributes to the unique characteristics of PMF (Kellens et al., 2007; Calliauw et al., 2007; Son et al., 2010; Hashimoto et al., 2001). POP level is usually set as more than $40 \%$ to qualify as sPMF. The percentage of diunsaturated triacylglycerols ranged from $14.33 \%$ to $30.57 \%$ and triunsaturated triacylglycerols from $3.19 \%$ to $5.16 \%$. The percentage of trisaturated triacylglycerols detected in this category was very low, ranging from $2.45 \%$ to $5.41 \%$. This combination of triacylglycerols with a very high ratio of unsaturated triacylglycerols to 
TABLE 1. TRIACYLGLYCEROL COMPOSITION OF SOFT PALM MID FRACTIONS

\begin{tabular}{|c|c|c|c|c|c|c|c|c|}
\hline & sPMF 1 & sPMF 2 & sPMF 3 & sPMF 4 & sPMF 5 & sPMF 6 & sPMF 7 & sPMF 8 \\
\hline LLL & $0.30 \pm 0.02$ & $0.30 \pm 0.01$ & $0.46 \pm 0.09$ & $0.30 \pm 0.07$ & $0.22 \pm 0.11$ & $0.23 \pm 0.07$ & $0.15 \pm 0.04$ & $0.25 \pm 0.04$ \\
\hline PLL & $1.87 \pm 0.01$ & $1.74 \pm 0.31$ & $1.68 \pm 0.06$ & $1.41 \pm 0.07$ & $1.37 \pm 0.10$ & $1.57 \pm 0.16$ & $1.39 \pm 0.23$ & $1.37 \pm 0.12$ \\
\hline MLP & $0.51 \pm 0.03$ & $0.58 \pm 0.19$ & $0.34 \pm 0.02$ & $0.37 \pm 0.05$ & $0.51 \pm 0.06$ & $0.61 \pm 0.14$ & $0.72 \pm 0.03$ & $0.40 \pm 0.17$ \\
\hline OOL & $1.34 \pm 0.08$ & $1.34 \pm 0.13$ & $1.17 \pm 0.02$ & $0.96 \pm 0.16$ & $1.11 \pm 0.04$ & $0.88 \pm 0.04$ & $0.99 \pm 0.02$ & $0.78 \pm 0.04$ \\
\hline POL & $7.66 \pm 0.29$ & $6.96 \pm 0.11$ & $5.99 \pm 0.04$ & $5.52 \pm 0.09$ & $5.97 \pm 0.02$ & $5.52 \pm 0.01$ & $4.94 \pm 0.01$ & $4.73 \pm 0.18$ \\
\hline PLP & $9.02 \pm 0.48$ & $8.90 \pm 0.08$ & $8.72 \pm 0.06$ & $8.26 \pm 0.24$ & $9.01 \pm 0.20$ & $8.38 \pm 0.26$ & $9.03 \pm 0.05$ & $7.31 \pm 0.15$ \\
\hline MPP & $1.76 \pm 0.18$ & $1.82 \pm 0.07$ & $2.00 \pm 0.04$ & $2.13 \pm 0.11$ & $1.94 \pm 0.08$ & $1.95 \pm 0.07$ & $2.35 \pm 0.01$ & $1.65 \pm 0.03$ \\
\hline $\mathrm{OOO}$ & $3.52 \pm 0.54$ & $2.98 \pm 0.06$ & $2.72 \pm 0.00$ & $2.58 \pm 0.30$ & $2.42 \pm 0.15$ & $2.79 \pm 0.19$ & $2.23 \pm 0.03$ & $2.16 \pm 0.31$ \\
\hline POO & $19.29 \pm 0.48$ & $16.59 \pm 0.01$ & $14.58 \pm 0.11$ & $15.72 \pm 0.09$ & $14.73 \pm 0.05$ & $15.75 \pm 0.08$ & $12.28 \pm 0.09$ & $11.90 \pm 0.07$ \\
\hline POP & $43.53 \pm 0.19$ & $45.65 \pm 0.12$ & $48.27 \pm 0.40$ & $49.20 \pm 0.16$ & $49.33 \pm 0.24$ & $49.64 \pm 0.16$ & $51.56 \pm 0.22$ & $55.95 \pm 0.10$ \\
\hline РPP & $0.69 \pm 0.04$ & $2.37 \pm 0.04$ & $2.26 \pm 0.05$ & $1.44 \pm 0.01$ & $1.49 \pm 0.20$ & $1.55 \pm 0.02$ & $2.57 \pm 0.04$ & $0.76 \pm 0.04$ \\
\hline SOO & $1.73 \pm 0.06$ & $1.50 \pm 0.03$ & $1.52 \pm 0.08$ & $1.39 \pm 0.07$ & $1.59 \pm 0.18$ & $1.42 \pm 0.05$ & $1.05 \pm 0.05$ & $1.06 \pm 0.07$ \\
\hline POS & $7.73 \pm 0.10$ & $7.84 \pm 0.06$ & $8.69 \pm 0.05$ & $9.32 \pm 0.49$ & $9.12 \pm 0.03$ & $8.48 \pm 0.02$ & $9.23 \pm 0.05$ & $10.46 \pm 0.01$ \\
\hline PPS & $0.00 \pm 0.00$ & $0.55 \pm 0.00$ & $0.45 \pm 0.02$ & $0.35 \pm 0.04$ & $0.00 \pm 0.00$ & $0.32 \pm 0.01$ & $0.48 \pm 0.02$ & $0.00 \pm 0.00$ \\
\hline SOS & $1.03 \pm 0.04$ & $0.87 \pm 0.10$ & $1.12 \pm 0.05$ & $1.06 \pm 0.11$ & $1.20 \pm 0.01$ & $0.90 \pm 0.04$ & $1.02 \pm 0.11$ & $1.21 \pm 0.03$ \\
\hline PSS & $0.00 \pm 0.00$ & $0.00 \pm 0.00$ & $0.00 \pm 0.00$ & $0.00 \pm 0.00$ & $0.00 \pm 0.00$ & $0.00 \pm 0.00$ & $0.00 \pm 0.00$ & $0.00 \pm 0.00$ \\
\hline SSS & $2.45 \pm 0.22$ & $4.75 \pm 0.12$ & $4.72 \pm 0.12$ & $3.91 \pm 0.06$ & $3.44 \pm 0.29$ & $3.83 \pm 0.10$ & $5.41 \pm 0.03$ & $2.41 \pm 0.07$ \\
\hline SUS & $61.83 \pm 0.15$ & $63.85 \pm 0.16$ & $67.15 \pm 0.11$ & $68.21 \pm 0.13$ & $69.16 \pm 0.06$ & $68.01 \pm 0.06$ & $71.55 \pm 0.17$ & $80.07 \pm 0.13$ \\
\hline SUU & $30.56 \pm 0.23$ & $26.79 \pm 0.17$ & $23.77 \pm 0.07$ & $24.04 \pm 0.13$ & $23.66 \pm 0.35$ & $24.25 \pm 0.18$ & $19.66 \pm 0.27$ & $14.33 \pm 0.12$ \\
\hline UUU & $5.16 \pm 0.44$ & $4.61 \pm 0.10$ & $4.36 \pm 0.11$ & $3.84 \pm 0.21$ & $3.74 \pm 0.00$ & $3.91 \pm 0.15$ & $3.37 \pm 0.02$ & $3.19 \pm 0.23$ \\
\hline
\end{tabular}

Note: sPMF - soft palm mid fraction, SSS - trisaturated triacylglycerol, SUS - monounsaturated triacylglycerol, SUU - diunsaturated triacylglycerol, UUU - triunsaturated triacylglycerol, M - myristic, P - palmitic, S - stearic, O - oleic, L- linoleic.

Each value in the table represents the mean \pm standard deviation of triplicate analyses $(n=3)$.

saturated triacylglycerols influenced the DP of these sPMF. These fractions are considered to be medium melting solid fractions by food formulators at the application temperature of $20^{\circ} \mathrm{C}$ to $30^{\circ} \mathrm{C}$ (Braipson and Gibon, 2007).

The four sPOs samples collected from the Malaysian fractionation plants were by-products from the production of palm olein IV 60 via single fractionation of palm oil. The monounsaturated triacylglycerols ranged from $55.56 \%$ to $57.33 \%$, with POP content ranging from $38.36 \%$ to $41.81 \%$. The percentage of diunsaturated ranged from $20.07 \%$ to $28.70 \%$ and triunsaturated triacylglycerols ranged from $4.28 \%$ to $6.28 \%$. The trisaturated triacylglycerols ranged from $10.46 \%$ to $18.52 \%$ with $6.31 \%$ to $13.88 \%$ of tripalmitoyl-glycerol (PPP), as shown in Table 2.

A significant difference was observed $(\mathrm{p}<0.02)$ among the monounsaturated triacylglycerols and POP values in SPMF and sPOs as shown in Table 3. Monounsaturated triacylglycerols were $4.5 \%$ to $24.51 \%$ lesser in sPOs than in sPMF. The amount of POP were $1.68 \%$ to $17.35 \%$ lesser in sPOs than the values detected in sPMF. Significant difference $(p<0.02)$ was observed among the trisaturated triacylglycerols and PPP values in sPMF and sPOs as shown in Table 3. The percentage of trisaturated triacylglycerols were very much higher in sPOs, approximately $5.41 \%$ to $16.07 \%$ higher than detected in SPMF. The triacylglycerol compositions of sPMF differed from the compositions of sPOs. Hence, triacylglycerol values can be used to differentiate these two fractions.

\section{Fatty Acid Composition}

The FAC of SPMF in Table 4 shows that the percentages of saturated fatty acids were the highest, ranging from $51.91 \%$ to $58.29 \%$ with $45.89 \%$ to $51.55 \%$ palmitic acid. The percentages of monounsaturated fatty acids which consisted mostly of oleic acid were $34.38 \%$ to $39.03 \%$. The percentages of polyunsaturated fatty acids ranged from $6.24 \%$ to $8.90 \%$. The percentages of saturated fatty acids were the highest in sPOs ranging from $56.19 \%$ to $63.08 \%$ with $49.74 \%$ to $56.57 \%$ of palmitic acid as shown in Table 5 . The percentages of monounsaturated fatty acids which consisted mostly of oleic acid were $30.98 \%$ to $35.57 \%$. The percentages of polyunsaturated fatty acids were in the range of $5.75 \%$ to $8.04 \%$.

No significant difference $(\mathrm{p}<0.02)$ was observed among the palmitic and oleic fatty acids in sPMF and sPOs as shown in Table 3. The FAC of sPMF and sPOs have overlapping ranges; the percentage of palmitic acid in sPMF was $45.89 \%$ to $51.55 \%$ and sPOs was $49.74 \%$ to $56.57 \%$. In the case of oleic acid, it was $34.49 \%$ to $38.88 \%$ in sPMF and $30.41 \%$ to $35.41 \%$ in sPOs. It is noteworthy that the arrangement of the fatty acids in the triacylglycerols in SPMF and sPOs varied substantially. The arrangement of the fatty acids in the triacylglycerol structure to a great extent determines the characteristics of the fats. Hence, in differentiating of sPMF from sPOs consideration of triacylglycerol composition will be a better reference as compared against FAC. 
TABLE 2. TRIACYLGLYCEROL COMPOSITION OF SOFT PALM STEARIN

\begin{tabular}{lcccc}
\hline & sPOs 1 & sPOs 2 & sPOs 3 & sPOs 4 \\
\hline LLL & $0.24 \pm 0.03$ & $0.66 \pm 0.20$ & $0.23 \pm 0.01$ & $0.19 \pm 0.04$ \\
PLL & $1.62 \pm 0.19$ & $1.71 \pm 0.24$ & $1.44 \pm 0.05$ & $1.33 \pm 0.11$ \\
MLP & $0.51 \pm 0.03$ & $1.07 \pm 0.05$ & $0.36 \pm 0.02$ & $0.46 \pm 0.07$ \\
OOL & $1.30 \pm 0.02$ & $1.83 \pm 0.22$ & $1.09 \pm 0.12$ & $0.87 \pm 0.10$ \\
POL & $7.33 \pm 0.33$ & $6.55 \pm 0.50$ & $5.94 \pm 0.11$ & $4.69 \pm 0.14$ \\
PLP & $8.43 \pm 0.23$ & $7.63 \pm 0.25$ & $7.80 \pm 0.06$ & $7.21 \pm 0.14$ \\
MPP & $1.70 \pm 0.05$ & $1.92 \pm 0.02$ & $1.57 \pm 0.02$ & $1.64 \pm 0.04$ \\
OOO & $3.8 \pm 0.02$ & $3.79 \pm 0.29$ & $3.54 \pm 0.07$ & $3.22 \pm 0.17$ \\
POO & $18.14 \pm 0.07$ & $15.59 \pm 0.24$ & $14.86 \pm 0.08$ & $12.99 \pm 0.38$ \\
POP & $39.77 \pm 0.22$ & $38.36 \pm 0.25$ & $38.61 \pm 0.02$ & $41.81 \pm 0.03$ \\
PPP & $6.31 \pm 0.18$ & $9.32 \pm 0.01$ & $13.39 \pm 0.03$ & $13.88 \pm 0.16$ \\
SOO & $1.62 \pm 0.02$ & $1.54 \pm 0.19$ & $1.29 \pm 0.02$ & $1.06 \pm 0.06$ \\
POS & $6.19 \pm 0.04$ & $6.51 \pm 0.14$ & $6.25 \pm 0.02$ & $6.96 \pm 0.07$ \\
PPS & $2.05 \pm 0.01$ & $2.35 \pm 0.12$ & $2.66 \pm 0.04$ & $2.73 \pm 0.08$ \\
SOS & $0.66 \pm 0.11$ & $0.72 \pm 0.08$ & $0.65 \pm 0.02$ & $0.88 \pm 0.12$ \\
PSS & $0.39 \pm 0.05$ & $0.44 \pm 0.01$ & $0.33 \pm 0.05$ & $0.27 \pm 0.02$ \\
\hline SSS & $10.46 \pm 0.09$ & $14.03 \pm 0.13$ & $17.94 \pm 0.03$ & $18.52 \pm 0.30$ \\
SUS & $55.56 \pm 0.13$ & $56.00 \pm 1.28$ & $53.68 \pm 0.10$ & $57.33 \pm 0.28$ \\
SUU & $28.70 \pm 0.13$ & $23.69 \pm 0.21$ & $23.53 \pm 0.04$ & $20.07 \pm 0.35$ \\
UUU & $5.41 \pm 0.01$ & $6.28 \pm 0.26$ & $4.86 \pm 0.17$ & $4.28 \pm 0.23$ \\
\hline NOte: & 504 & &
\end{tabular}

Note: sPOs - soft palm stearin, SSS - trisaturated triacylglycerol, SUS - monounsaturated triacylglycerol, SUU - diunsaturated triacylglycerol, UUU - triunsaturated triacylglycerol, M - myristic, P - palmitic, S - stearic, $\mathrm{O}$ - oleic, L - linoleic.

Each value in the table represents the mean \pm standard deviation of triplicate analyses $(n=3)$.

TABLE 3. QUALITY PARAMETRS OF SOFT PALM MID FRACTION AND SOFT PALM STEARIN

\begin{tabular}{llr}
\hline & sPMF & sPOs \\
\hline Triacylglycerols (TAG) & & $15.24 \pm 3.75^{\mathrm{b}}$ \\
$\quad$ Trisaturated TAG & $3.87 \pm 1.08^{\mathrm{a}}$ & $10.73 \pm 3.58^{\mathrm{b}}$ \\
$\quad$ Tripalmitoyl-glycerol & $1.64 \pm 0.71^{\mathrm{a}}$ & $55.64 \pm 1.51^{\mathrm{b}}$ \\
$\quad$ Monounsaturated TAG & $68.73 \pm 5.49^{\mathrm{a}}$ & $39.64 \pm 1.57^{\mathrm{b}}$ \\
1,3-dipalmitoyl-2-oleoyl-glycerol & $49.14 \pm 3.72^{\mathrm{a}}$ & $45.80 \pm 4.91^{\mathrm{b}}$ \\
Dropping point & $28.58 \pm 3.23^{\mathrm{a}}$ & \\
Fatty acid composition & & $53.17 \pm 2.94^{\mathrm{a}}$ \\
Palmitic acid & $49.59 \pm 1.82^{\mathrm{a}}$ & $32.93 \pm 2.20^{\mathrm{a}}$ \\
Oleic acid & $36.20 \pm 1.66^{\mathrm{a}}$ & $41.34 \pm 3.56^{\mathrm{a}}$ \\
Iodine value & $44.79 \pm 2.53^{\mathrm{a}}$ & \\
\hline
\end{tabular}

Note: $S$ - saturated, sPMF - soft palm mid fraction, sPOs - soft palm stearin.

For each quality parameter, means within each column with same superscripted letter ${ }^{\mathrm{a}-\mathrm{b}}$ are not significantly different at $\mathrm{p}=0.02$.

\section{Iodine Value}

Iodine values are directly related to FAC of the oils or fats. The increase in the amount of saturated fatty acids, will in turn decrease the iodine values of the fractions. The iodine values of sPMF ranged from 40.91 to 49.50 as shown in Table 4. sPMF 7 recorded the lowest IV of 40.91, consisted of $58.07 \%$ saturated, $35.54 \%$ monounsaturated and polyunsaturated $6.24 \%$ fatty acids. In contrast, sPMF 1 with the highest IV of 49.50, consisted of higher amount of monounsaturated and polyunsaturated fatty acids, the composition was
$51.91 \%$ saturated, $39.03 \%$ monounsaturated and $8.90 \%$ polyunsaturated fatty acids. The iodine values of sPOs ranged from 37.05 to 45.69 as shown in Table 5. The iodine values of sPOs decreased as the amount of saturated fatty acids increased and the amount of monounsaturated and polyunsaturated fatty acids decreased. sPOs 4 recorded the lowest IV of 37.05 , consisting of $63.08 \%$ of saturated fatty acids and sPOs 1 had the highest IV of 45.69 as it consisted the lowest amount of saturated fatty acids of $56.19 \%$. 
TABLE 4. FATTY ACID COMPOSITION, IODINE VALUE AND DROPPING POINT OF SOFT PALM MID FRACTIONS

\begin{tabular}{|c|c|c|c|c|c|c|c|c|}
\hline & sPMF 1 & sPMF 2 & sPMF 3 & sPMF 4 & sPMF 5 & sPMF 6 & sPMF 7 & sPMF 8 \\
\hline C12:0 & $0.17 \pm 0.00$ & $0.17 \pm 0.00$ & $0.33 \pm 0.04$ & $0.26 \pm 0.00$ & $0.20 \pm 0.00$ & $0.15 \pm 0.00$ & $0.44 \pm 0.05$ & $0.13 \pm 0.00$ \\
\hline C14:0 & $0.96 \pm 0.00$ & $1.06 \pm 0.00$ & $0.76 \pm 0.12$ & $1.08 \pm 0.00$ & $1.02 \pm 0.00$ & $1.00 \pm 0.00$ & $1.12 \pm 0.08$ & $0.84 \pm 0.01$ \\
\hline C16:0 & $45.89 \pm 0.06$ & $48.79 \pm 0.03$ & $50.64 \pm 0.52$ & $49.78 \pm 0.06$ & $49.17 \pm 0.02$ & $49.38 \pm 0.08$ & $51.53 \pm 0.04$ & $51.55 \pm 0.41$ \\
\hline C16:1 & $0.15 \pm 0.01$ & $0.14 \pm 0.00$ & $0.81 \pm 0.14$ & $0.12 \pm 0.00$ & $0.12 \pm 0.01$ & $0.13 \pm 0.01$ & $0.54 \pm 0.61$ & $0.10 \pm 0.00$ \\
\hline C18:0 & $4.52 \pm 0.01$ & $4.57 \pm 0.01$ & $5.15 \pm 0.04$ & $4.87 \pm 0.01$ & $4.90 \pm 0.00$ & $4.55 \pm 0.01$ & $4.83 \pm 0.31$ & $5.17 \pm 0.04$ \\
\hline C18:1 & $38.88 \pm 0.05$ & $36.92 \pm 0.07$ & $33.57 \pm 0.63$ & $36.36 \pm 0.04$ & $36.74 \pm 0.02$ & $37.16 \pm 0.01$ & $34.49 \pm 0.40$ & $35.44 \pm 0.51$ \\
\hline C18:2 & $8.64 \pm 0.00$ & $7.66 \pm 0.04$ & $7.54 \pm 0.03$ & $6.61 \pm 0.01$ & $7.16 \pm 0.01$ & $6.94 \pm 0.07$ & $6.47 \pm 0.46$ & $6.13 \pm 0.02$ \\
\hline C18:3 & $0.25 \pm 0.09$ & $0.16 \pm 0.00$ & $0.76 \pm 0.16$ & $0.09 \pm 0.00$ & $0.13 \pm 0.00$ & $0.11 \pm 0.00$ & $0.08 \pm 0.00$ & $0.11 \pm 0.20$ \\
\hline C20:0 & $0.38 \pm 0.00$ & $0.36 \pm 0.00$ & $0.35 \pm 0.01$ & $0.37 \pm 0.00$ & $0.38 \pm 0.00$ & $0.38 \pm 0.00$ & $0.37 \pm 0.02$ & $0.39 \pm 0.01$ \\
\hline Others & $0.16 \pm 0.02$ & $0.17 \pm 0.00$ & $0.10 \pm 0.00$ & $0.46 \pm 0.00$ & $0.16 \pm 0.00$ & $0.19 \pm 0.02$ & $0.15 \pm 0.02$ & $0.15 \pm 0.01$ \\
\hline Saturated & $51.91 \pm 0.05$ & $54.95 \pm 0.04$ & $57.22 \pm 0.63$ & $56.35 \pm 0.05$ & $55.67 \pm 0.025$ & $55.47 \pm 0.09$ & $58.29 \pm 0.51$ & $58.07 \pm 0.47$ \\
\hline Monounsaturated & $39.03 \pm 0.04$ & $37.06 \pm 0.08$ & $34.38 \pm 0.77$ & $36.48 \pm 0.04$ & $36.87 \pm 0.013$ & $37.30 \pm 0.02$ & $35.04 \pm 1.00$ & $35.54 \pm 0.51$ \\
\hline Polyunsaturated & $8.90 \pm 0.00$ & $7.82 \pm 0.04$ & $8.30 \pm 0.13$ & $6.70 \pm 0.01$ & $7.30 \pm 0.011$ & $7.05 \pm 0.07$ & $6.54 \pm 0.47$ & $6.24 \pm 0.03$ \\
\hline Others & $0.16 \pm 0.02$ & $0.17 \pm 0.00$ & $0.10 \pm 0.00$ & $0.46 \pm 0.00$ & $0.16 \pm 0.01$ & $0.19 \pm 0.02$ & $0.15 \pm 0.02$ & $0.15 \pm 0.01$ \\
\hline Iodine value $\left(\mathrm{I}_{2} / 100 \mathrm{~g}\right)$ & $49.50 \pm 0.13$ & $45.83 \pm 0.23$ & $45.98 \pm 0.09$ & $43.27 \pm 0.08$ & $44.38 \pm 0.17$ & $45.18 \pm 0.08$ & $40.91 \pm 0.20$ & $43.26 \pm 0.12$ \\
\hline Dropping point $\left({ }^{\circ} \mathrm{C}\right)$ & $23.4 \pm 0.21$ & $31.6 \pm 0.14$ & $31.8 \pm 0.10$ & $28.3 \pm 0.21$ & $28.0 \pm 0.14$ & $27.6 \pm 0.14$ & $32.5 \pm 0.10$ & $25.4 \pm 0.14$ \\
\hline
\end{tabular}

Note: sPMF - soft palm mid fraction.

Each value in the table represents the mean \pm standard deviation of triplicate analyses $(n=3)$.

TABLE 5. FATTY ACID COMPOSITION, IODINE VALUE AND DROPPING POINT OF SOFT PALM STEARINS

\begin{tabular}{lccc}
\hline & sPOs 1 & sPOs 2 & sPOs 3 \\
\hline C12:0 & $0.19 \pm 0.00$ & $0.19 \pm 0.01$ & $0.68 \pm 0.01$ \\
C14:0 & $1.22 \pm 0.00$ & $1.84 \pm 0.00$ & $1.17 \pm 0.02$ \\
C16:0 & $49.74 \pm 0.05$ & $52.04 \pm 0.01$ & $54.33 \pm 0.47$ \\
C16:1 & $0.15 \pm 0.02$ & $0.13 \pm 0.00$ & $0.49 \pm 0.54$ \\
C18:0 & $4.66 \pm 0.00$ & $5.09 \pm 0.01$ & $4.86 \pm 0.02$ \\
C18:1 & $35.41 \pm 0.01$ & $32.16 \pm 0.02$ & $31.23 \pm 0.22$ \\
C18:2 & $7.79 \pm 0.00$ & $6.64 \pm 0.00$ & $6.72 \pm 0.14$ \\
C18:3 & $0.25 \pm 0.00$ & $0.10 \pm 0.00$ & $0.10 \pm 0.01$ \\
C20:0 & $0.38 \pm 0.00$ & $0.37 \pm 0.00$ & $0.31 \pm 0.02$ \\
Others & $0.21 \pm 0.05$ & $0.47 \pm 0.00$ & $0.11 \pm 0.02$ \\
\hline Saturated & $56.19 \pm 0.06$ & $59.53 \pm 0.00$ & $61.35 \pm 0.43$ \\
Monounsaturated & $35.57 \pm 0.03$ & $33.28 \pm 0.01$ & $31.72 \pm 0.32$ \\
Polyunsaturated & $8.04 \pm 0.01$ & $6.74 \pm 0.01$ & $6.82 \pm 0.13$ \\
Others & $0.21 \pm 0.05$ & $0.47 \pm 0.00$ & $0.11 \pm 0.02$ \\
\hline Iodine value $\left(\mathrm{I}_{2} / 100 \mathrm{~g}\right)$ & $45.69 \pm 0.08$ & $41.93 \pm 0.15$ & $4.00 \pm 0.00$ \\
\hline Dropping point $\left({ }^{\circ} \mathrm{C}\right)$ & $40.3 \pm 0.10$ & $42.5 \pm 0.10$ & $0.05 \pm 0.07$ \\
\hline Note: & & $40.68 \pm 0.15$ & $0.20 \pm 0.00$ \\
\hline
\end{tabular}

Note: sPOs - soft palm stearin.

Each value in the table represents the mean \pm standard deviation of triplicate analyses $(n=3)$.

The iodine values reflect the composition of the fatty acids, hence, it is not an advisable comparison parameter to differentiate between sPMF and sPOs. The iodine values of sPMF and sPOs was not significantly different, sPMF with 40.91 to 49.50 and sPOs with 37.05 to 45.69 . No significant difference $(\mathrm{p}<0.02)$ was observed among the iodine values in sPMF and sPOs (Table 3). Iodine values are not able to reflect the actual characteristics of these two very varied fractions. IV determines the level of unsaturation of the fatty acids and independent of the arrangement or position of the fatty acids in the triacylglycerol structure. Hence, IV should not be considered as a quality parameter to differentiate these two fractions.

\section{Dropping Point}

The DP of sPMF ranged from $23.4^{\circ} \mathrm{C}$ to $32.5^{\circ} \mathrm{C}$ as shown in Table 4. The variation in the DP is attributed to the triacylglycerol composition of these fractions. The low amount of trisaturated triacylglycerols in sPMF $1(2.45 \%)$ and sPMF 8 $(2.41 \%)$, resulted in lower DP values of $23.4^{\circ} \mathrm{C}$ and $25.4^{\circ} \mathrm{C}$, respectively. In contrast, sPMF 2 and sPMF 3 with higher amount of trisaturated triacylglycerols 
of $4.75 \%$ and $4.72 \%$, gave higher DP values of $31.6^{\circ} \mathrm{C}$ and $31.8^{\circ} \mathrm{C}$, respectively. The DP of sPOs varied between $40.3^{\circ} \mathrm{C}$ to $50.1^{\circ} \mathrm{C}$ as shown in Table 5. The high DP values of sPOs were caused by the higher amount of trisaturated triacylglycerols present in the fractions. Sample sPOs 1 contained $10.46 \%$ of trisaturated triacylglycerols which gave a DP value of $40.3^{\circ} \mathrm{C}$ and sPOs 4 with $18.52 \%$ of trisaturated triacylglycerols gave a higher DP value of $50.1^{\circ} \mathrm{C}$. Although the IV overlapped in the range of 40 to 46 between the two types of palm solid fractions, the DP varied distinctively. The DP of sPMF ranged between $24.3^{\circ} \mathrm{C}$ to $31.8^{\circ} \mathrm{C}$ and sPOs varied between $40.3^{\circ} \mathrm{C}$ to $50.1^{\circ} \mathrm{C}$, a substantial difference of $8.5^{\circ} \mathrm{C}$ to $25.8^{\circ} \mathrm{C}$. This difference is due to the varied triacylglycerol compositions in the fractions. DP values were able to express the physical variation between sPMF and sPOs. The DP values obtained for these fats were high ranging from $40.3^{\circ} \mathrm{C}$ to $50.1^{\circ} \mathrm{C}$. Similar values were reported by Rashid et al. of palm stearin with IV of 40.8 gave an slip melting point of $49.6^{\circ} \mathrm{C}$ (Rashid et al., 2012).

Significant difference $(p<0.02)$ was observed among the DP values in SPMF and sPOs as shown in Table 3. DP of sPMF ranged from $23.4^{\circ} \mathrm{C}$ to $32.5^{\circ} \mathrm{C}$, in contrast sPOs exhibited much higher DP values of $40.3^{\circ} \mathrm{C}$ to $50.1^{\circ} \mathrm{C}$. Hence, DP can be used to differentiate these two fractions.

\section{Solid Fat Content}

The SFC profiles of sPMF showed similar trends even though the triacylglycerol compositions differed (Figure 2). The SFC values of sPMF were from $48 \%$ to $65 \%$ at $15^{\circ} \mathrm{C}$ and from $34 \%$ to $51 \%$ at $20^{\circ} \mathrm{C}$. These fractions experienced a drastic decrease in SFC between $20^{\circ} \mathrm{C}$ to $25^{\circ} \mathrm{C}$ (a mere increase of $\left.5^{\circ} \mathrm{C}\right), \mathrm{SFC}$ decreased from $34 \%$ to $51 \%$ at $20^{\circ} \mathrm{C}$ to below $15 \%$ at $25^{\circ} \mathrm{C}$. The substantial difference in SFC was $15 \%$ to $35 \%$ between the temperatures of $20^{\circ} \mathrm{C}$ to $25^{\circ} \mathrm{C}$. This sharp decrease in the percentage of SFC values were in the range of $20^{\circ} \mathrm{C}$ to $25^{\circ} \mathrm{C}$, which denotes the melting of the monounsaturated triacylglycerols, which are a major component of sPMF. The percentage of monounsaturated triacylglycerols present in SPMF are from $61.83 \%$ to $80.07 \%$. SFC values were from $0.0 \%$ to $5 \%$ at $30^{\circ} \mathrm{C}$. Composition of triacylglycerols strongly influences the SFC profile patterns. The main monounsaturated triacylglycerol is POP $(46 \%$ to $52 \%)$, hence, the SFC

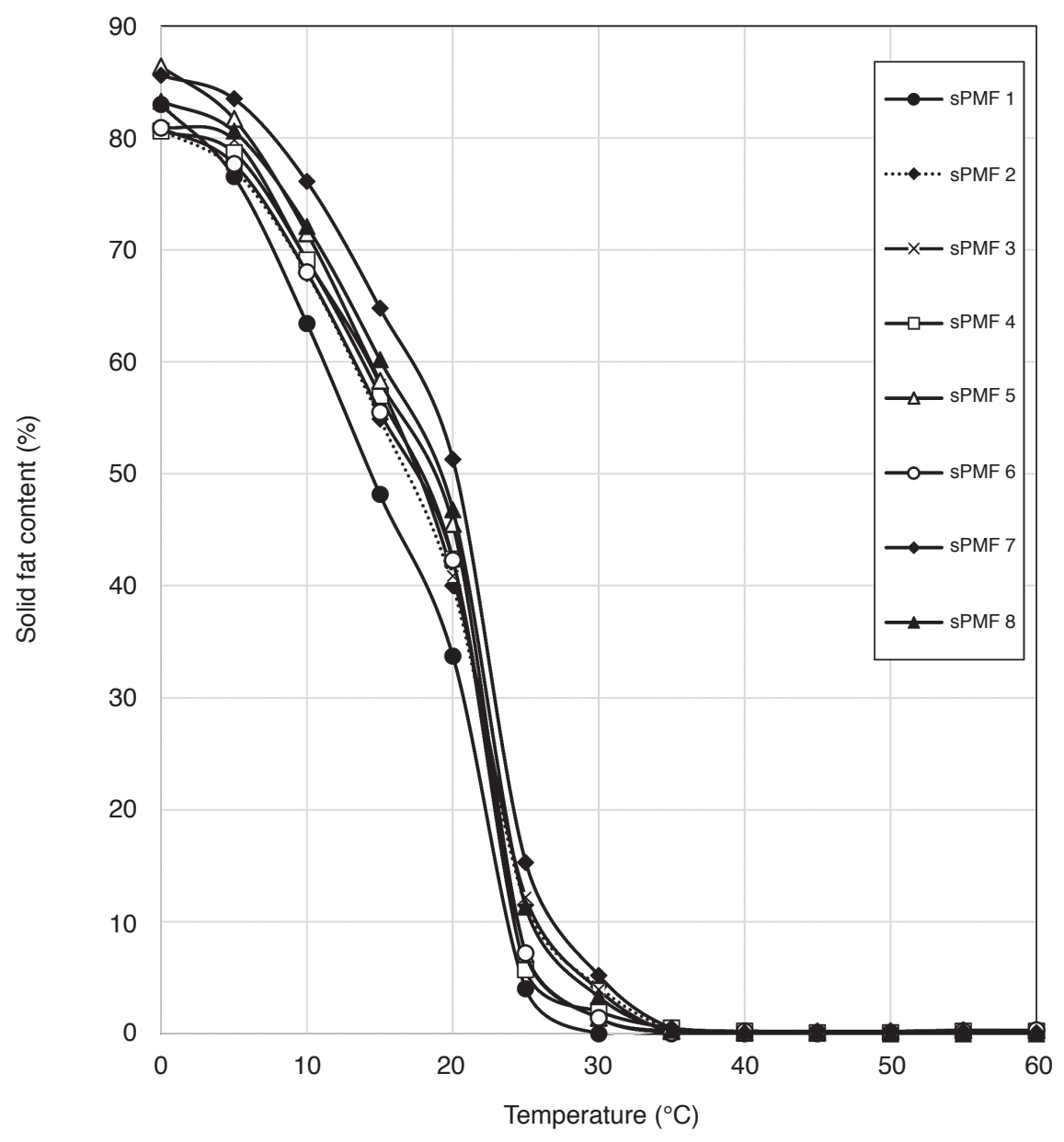

Figure 2. Solid fat content of soft palm mid fractions (sPMF). 
profile pattern was dominated by the characteristic of POP that has a DP of $30.5^{\circ} \mathrm{C}\left(\beta^{\prime}\right)$ to $35.5^{\circ} \mathrm{C}(\beta)$. This unique SFC profile is preferred by the chocolate industry, hence, it is widely used as a cocoa butter equivalent (Bootello et al., 2012). POP rich fats are usually blended with exotic crops such as shea, sal and illipe fats (source of palmitoyl--oleoyl-strearoylglycerol and distrearoyl -oleoyl-glycerol), to obtain fat blends that are compatible to cocoa butter.

The SFC profiles of sPOs varied in the degree of SFC present at individual temperatures reflecting the variation in the triacylglycerols present (Figure 3 ). The variation was due to the level of trisaturated triacylglycerols present, as the percentage of trisaturated triacylglycerols increased, the percentage of SFC at the respective temperatures increased. The SFC profiles of sPOs did not exhibit a sharp drop over the temperature range evaluated, rather a gradual decrease in SFC values was experienced with the increase in temperature. A decrease of $28 \%$ to $32 \%$ of SFC was observed between $20^{\circ} \mathrm{C}$ to $30^{\circ} \mathrm{C}$ (sPOs 1 dropped from $42 \%$ to $14 \%$, sPOs 2 from $49 \%$ to $20 \%$, sPOs 3 from $59 \%$ to $28 \%$ and sPOs 4 from $63 \%$ to $30 \%$ ). This drop in SFC was due to the melting of monounsaturated triacylglycerols which were $54 \%$ to $57 \%$ (POP $38 \%$ to $42 \%$ ) of the triacylglycerol composition. The percentage of SFC present at $45^{\circ} \mathrm{C}$ ranged from $0.3 \%$ to $10 \%$.

The SFC profile of SPMF and sPOs differed at the temperature range of $15^{\circ} \mathrm{C}$ to $30^{\circ} \mathrm{C}$. The point to emphasise is that sPOs did not mimic the SFC profiles of SPMF although they have a high percentage of POP but on the contrary, exhibited a 'tail effect' due to the presence of trisaturated triacylglycerols, that ranged from $10 \%$ to $18 \%$. Hence, SFC profiles for sPMF and sPOs can be established and used to differentiate these two fractions.

\section{Crystallisation and Melting Behaviour}

The crystallisation and melting curves of sPMF are as shown in Figure 4. The position of the peaks in the crystallisation and melting profiles are very much dominated by the triacylglycerol composition that is strongly related to the content of low melting, medium melting and high melting triacylglycerols (Braipson and Gibon, 2007; Marangoni and Lencki, 1998). The onset temperature of the crystallisation profile is characterised by the beginning of fat

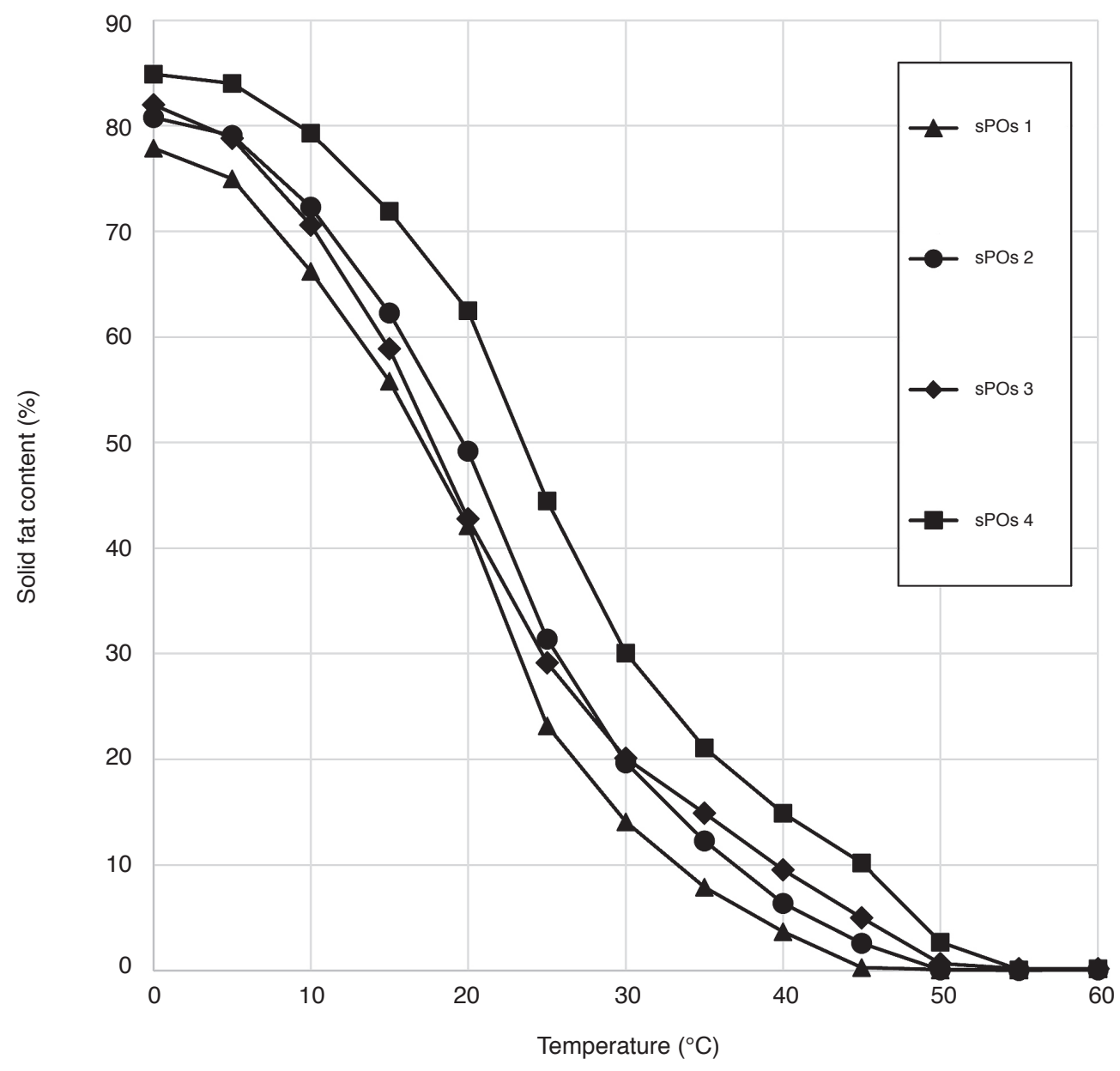

Figure 3. Solid fat content of soft palm stearins (sPOs). 

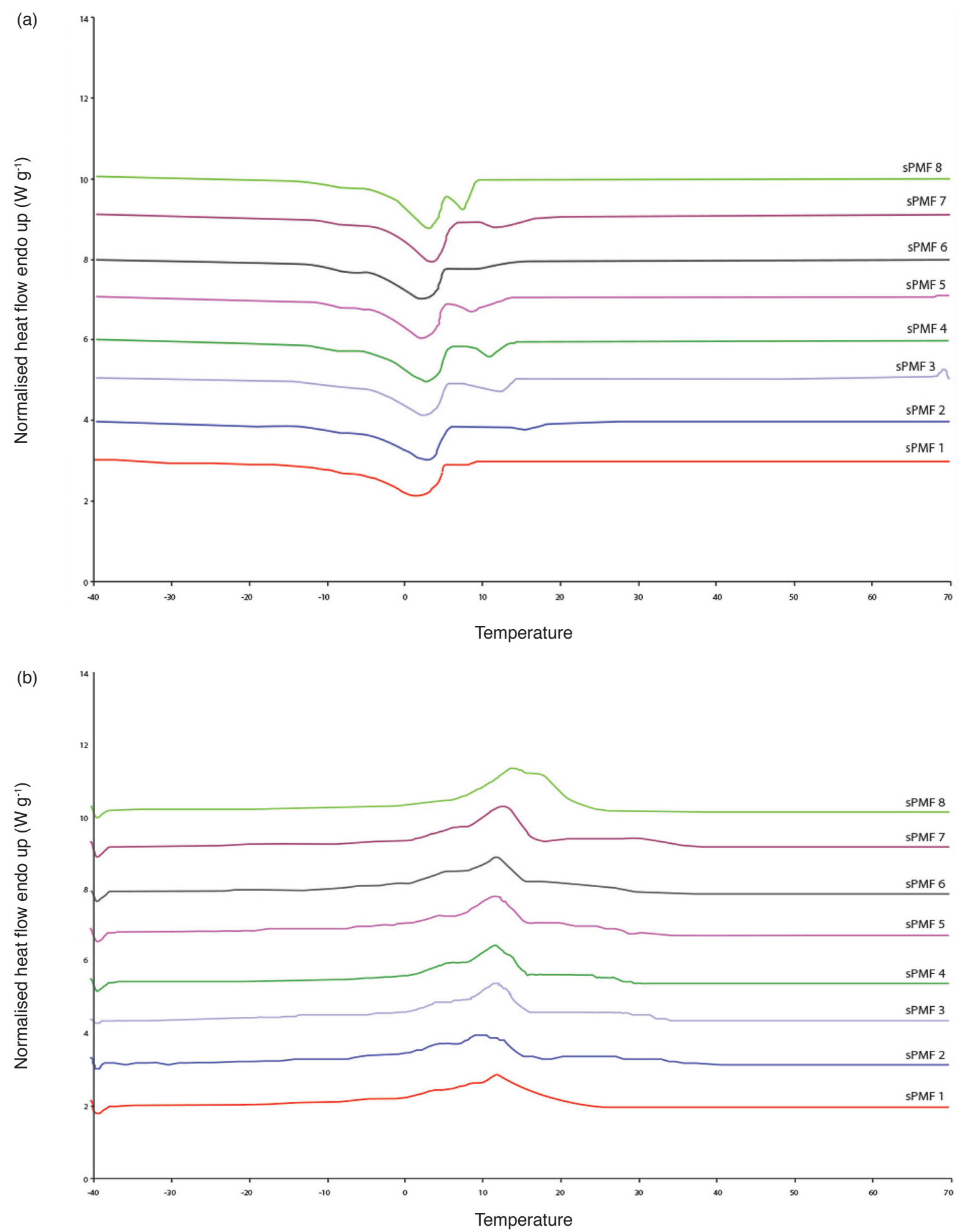

Figure 4. Differential scanning calorimetry (DSC) thermograms of (a) cooling (crystallisation) and (b) heating (melting) of soft palm mid fractions (sPMF).

crystal formation, which is greatly influenced by the content of saturated triacylglycerols combined with rapid cooling and the offset temperature explains the end of crystallisation (Saadi et al., 2012). The onset temperature of sPMF differed with the amount of saturated triacylglycerols present in the fractions.
Fraction sPMF 1 exhibited a single exothermic peak, displaying the homogenous crystallisation and formation of crystals. sPMF 2 to sPMF 8 exhibited a well-defined two stage crystallisation. The events indicated initial crystallisation of trisaturated triacylglycerols and continued with the crystallisation 
of monounsaturated and diunsaturated triacylglycerols. The offset crystallisation temperature of sPMF 1 to sPMF 8 were between $-29^{\circ} \mathrm{C}$ to $-31^{\circ} \mathrm{C}$, indicating the delayed crystallisation of triunsaturated triacylglycerols. The melting profiles of sPMF exhibited a major endothermic peak accompanied by a broad endothermic peak. sPMF 8 had the highest peak attributed by its high monounsaturated triacylglycerols level of $80 \%$ and this was closely followed by sPMF 7 with $71 \%$ of monounsaturated triacylglycerols. sPMF 3, sPMF 4, sPMF 5 and sPMF 6 exhibited a very similar pattern of endothermic melting peaks. This trend was aligned to the composition of monounsaturated triacylglycerols present in these fractions which varied from $67.15 \%$ to $68.01 \%$ with approximately $48.27 \%$ to $49.64 \%$ of POP. The lowest peak was exhibited by sPMF 1 , which was related to the lowest percentage of monounsaturated triacylglycerols of $61.83 \%$ with $43.53 \%$ of POP. sPMF 1 and sPMF 8 had only one endothermic peak present without a tailing broad peak. These fractions had low percentage of trisaturated triacylglycerols of $2.4 \%$, hence, the endothermic broad peak indicating the crystallisation of these trisaturated triacylglycerols were not evident as compared to SPMF 2 to sPMF 7. sPMF 2 and sPMF 7 had $3.44 \%$ to $5.41 \%$ of trisaturated triacylglycerols, with $2.4 \%$ and $2.6 \%$ of tripalmitin (PPP), respectively. Hence, sPMF 2 and sPMF 7 showed broader secondary endothermic peaks indicating melting of high melting trisaturated triacylglycerols. Similar observations were reported in binary mixtures of $\mathrm{PPP} / \mathrm{POP}$ with a blending ratio of 10:90. It was reported that the lowering and broadening of peaks denoting the melting behaviour of PPP is caused by level of solubility of PPP in the presence of liquid POP (Minato et al., 1996). These phenomena also explain the complete melting of PPP at temperatures below $40^{\circ} \mathrm{C}$.

The sPOs crystallisation profile exhibited two exothermic peaks, as shown in Figure 5. The onset temperature of the first exothermic peak varied with the percentage of trisaturated triacylglycerols. As the percentage of trisaturated triacylglycerols increased, the onset temperature increased. The lowest onset temperature was exhibited by sPOs 1 at $18^{\circ} \mathrm{C}$ with $10 \%$ trisaturated triacylglycerols, followed by sPOs 2 at $22^{\circ} \mathrm{C}$ with $14 \%$ trisaturated triacylglycerols. The highest onset temperature was by sPOs 3 and sPOs 4 at $24^{\circ} \mathrm{C}$ with $18 \%$ trisaturated triacylglycerols. The sPOs were crystallised rapidly at the rate of $10^{\circ} \mathrm{C}$ $\min ^{-1}$, hence, crystallised instantaneously. High melting triacylglycerols were rapidly cooled which initiated rapid crystallisation, forming solids within a liquid phase. This occurrence was accompanied by a rapid increase in viscosity, thus, severely limiting mass transfer, hence, a single major peak was observed representing the combined crystallisation of trisaturated, monounsaturated and diunsaturated triacylglycerols (Campos et al., 2002; Toro-Vazquez et al., 2001). The second endothermic peak was a broad peak stretching from approximately $5^{\circ} \mathrm{C}$ to $-30^{\circ} \mathrm{C}$, indicating the combined crystallisation of low and medium melting triacylglycerols.

The melting profiles of sPOs fractions presented in Figure 5 exhibit two significant endothermic peaks. The positions of the two endothermic peaks were, one below $20^{\circ} \mathrm{C}$ and the other above $20^{\circ} \mathrm{C}$ (Anais et al., 2010). The two endothermic peaks corresponded to melting transitions of low melting triacylglycerols (low temperature endotherm is from $-30^{\circ} \mathrm{C}$ to $20^{\circ} \mathrm{C}$ ) and the second peak indicating the melting of the medium melting and high melting triacylglycerols (medium and high temperature endotherm is from $20^{\circ} \mathrm{C}$ to $50^{\circ} \mathrm{C}$ ) (Anais et al., 2010; Tan and Che Man, 2002). In sPOs 3 and sPOs 4, a third endothermic peak was observed; which corresponded to the presence of higher amount of high melting saturated triacylglycerols. The amount of trisaturated triacylglycerols in sPOs 3 and sPOs 4 were $17.94 \%$ and $18.52 \%$, respectively as compared to sPOs 1 and sPOs 2 with $10.46 \%$ and $14.03 \%$, respectively.

The sPMF and sPOs exhibit unique and distinctive crystallisation and melting profiles. The crystallisation and melting profile reflect the triacylglycerol composition of the fractions. Hence, crystallisation and melting profile can be established and used to differentiate these two fractions.

\section{CONCLUSION}

Specific chemical and physical characteristics of sPMF differ from sPOs. sPMF contained higher amounts of monounsaturated triacylglycerols than sPOs. The monounsaturated triacylglycerols of sPMF ranged from $61.83 \%$ to $80.07 \%$ (POP from $43.53 \%$ to $55.95 \%$ ) and sPOs from $55.56 \%$ to $57.33 \%$ (POP from $38.36 \%$ to $41.81 \%$ ). The trisaturated triacylglycerols ranged from $2.45 \%$ to $5.41 \%$ in sPMF and $10.46 \%$ to $18.52 \%$ in sPOs. SFC profile of sPMF and sPOs varied significantly. The sPMF exhibited a sharp decrease in the percentage of solids from $20^{\circ} \mathrm{C}$ to $30^{\circ} \mathrm{C}$. The SFC values of these fractions were $34 \%$ to $51 \%$ at $20^{\circ} \mathrm{C}$ and below $0.0 \%$ to $5 \%$ at $30^{\circ} \mathrm{C}$. In contrast, sPOs did not exhibit a sharp decrease between $20^{\circ} \mathrm{C}$ to $30^{\circ} \mathrm{C}$ temperature and had $0.3 \%$ to $10 \%$ of solids at $45^{\circ} \mathrm{C}$ giving the fraction a tailing effect. The DP of the fractions also varied significantly. The DP of sPMF ranged from $24.3^{\circ} \mathrm{C}$ to $31.8^{\circ} \mathrm{C}$ and that of sPOs ranged from $40.3^{\circ} \mathrm{C}$ to $50.1^{\circ} \mathrm{C}$. sPMF and sPOs exhibit unique and distinctive crystallisation and melting profiles which can be used to differentiate these two fractions. Thus, triacylglycerol composition, DP, SFC and thermal behaviour (crystallisation and melting) are indicative parameters that are able to assist in 

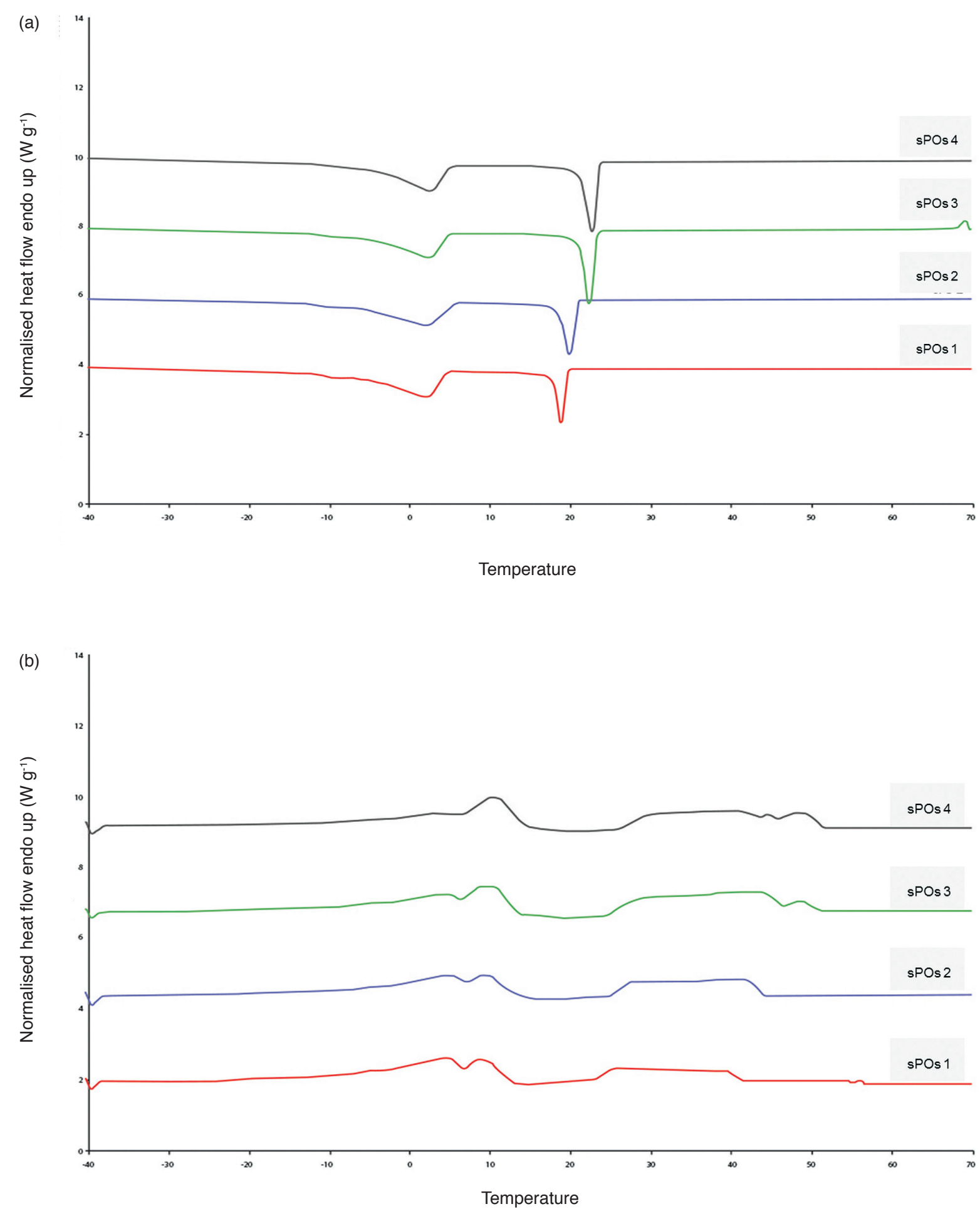

Figure 5. Differential scanning calorimetry (DSC) thermograms of (a) cooling (crystallisation) and (b) heating (melting) of soft palm stearins (SPOs).

determining the variations in the characteristics of sPMF and sPOs. In contrast, FAC and iodine values are objectionable indicators to differentiate these two solid fractions. The specification on the sPMF and sPOs should be addressed and established to avoid confusion in sales and purchase transactions.

\section{ACKNOWLEDGEMENT}

The authors would like to thank the management of MPOB for permission to execute and publish this work. The authors would also like to thank the staff of the Oils and Fats Technology Centre (OFTEC), 
MPOB - Zakaria Md Yusof, Nasoikheiddinah Md Purdi, Zukarinah Kamarudin, Hanirah Hassan, Adrina Malek, Che Maimon Che $\mathrm{Ha}$, Ramlah Ahmad, Zaimi Ibrahim, Abd Rahman Ibrahim and Rosnani Osman for their support. Appreciation is extended to Malini Devi of Sime Dardy Jomalina Sdn Bhd, Amy Shum of Sawit Raya Sdn Bhd, Beatrice Ting Thing Min of SOP Edible Oils Sdn Bhd, Rozi Pilos of Premium Vegetable Oil Sdn Bhd, Jimmy Loh Keng Leong of Moi Foods Malaysia Sdn Bhd, Dr Kyaw Zay Ya of Agri Asia Refinery Sdn Bhd, Lucy Lau of Mewah Oils Sdn Bhd and Lim Choh Hock of Ecolex Sdn Bhd for the in-depth discussion and information shared on the fractionation scenario in Malaysia.

\section{REFERENCES}

Adhikari, P; Zhu, X; Gautam, A; Shin, J; Lee, J; Akoh, C C and Lee, K (2010). Scaled-up production of zero-trans margarine fat using pine nut oil and palm stearin. Food Chemistry, 119(4): 1332-1338.

Anais, V; Lucy, K; Boulos, Y; Mathilde, B and Jean, M S (2010). Thermal and structural behavior of palm oil. Influence of cooling rate on fat crystallization. Macromolecular Symposia, 290: 137-145.

Biswas, N; Cheow, Y L; Tan, C P; Kanagaratnam, S and Siow, L F (2017). Cocoa butter substitute (CBS) produced from palm mid-fraction/palm kernel oil/ palm stearin for confectionery fillings. J. Amer. Oil Chem. Soc., 94(2): 1-11.

Bootello, M A; Hartel, R W; Garcés, R; MartínezForce, E and Salas, J J (2012). Evaluation of high oleic-high stearic sunflower hard stearins for cocoa butter equivalent formulation. Food Chemistry, 134: 1409-1417.

Braipson, D S and Gibon, V (2007). Comparative analysis of triacylglycerol composition, melting properties and polymorphic behavior of palm oil and fractions. European J. Lipid Science and Technology, 109: 359-372.

Calliauw, G; Gibon, V; De Greyt, W; Plees, L; Foubert, I and Dewettinck, K (2007). Phase composition during palm olein fractionation and its effect on soft PMF and superolein quality. J. Amer. Oil Chem. Soc., 84(9): 885-891.

Campos, R; Narine, S S and Marangoni, A G (2002). Effect of cooling rate on the structure and mechanical properties of milk fat and lard. Food Research International, 35: 971-981.

Danthine, S; Lefébure, E; Blecker, C; Dijckmans, P and Gibon, V (2017). Correlations between cloud point and compositional properties of palm oil and liquid fractions from dry fractionation. J. Amer. Oil Chem. Soc., 94(6): 841-853.

Gee, P T (2007). Analytical characteristics of crude and refined palm oil and fractions. European J. Lipid Science and Technology, 109: 373-379.

Gijs, C; Véronique, G; Wim, D G; Laurence, P; Imogen, $F$ and Koen, D (2007). Phase composition during palm olein fractionation and its effect on soft PMF and superolein quality. J. Amer. Oil Chem. Soc., 84(9): 885-891.

Hashem, H A A; Ellh, A N; Eltawab, A G and AbdelRazek, A G (2018). Industrial scale production of palm super olein using modified and innovative dry fractionation technique. Egyptian J. Chemistry, 61(1): $1-11$.

Hashimoto, S; Nezu, T; Arakawa, H; Ito, T and Maruzeni, S (2001). Preparation of sharp-melting hard palm mid-fraction and its use as hard butter in chocolate. J. Amer. Oil Chem. Soc., 78(5): 455-460.

Jahurul, M H A; Zaidul, I S M; Norulaini, N A N; Sahena, F; Abedin, M Z; Ghafoor, K and Mohd Omar, A K (2014). Characterization of crystallization and melting profiles of blends of mango seed fat and palm oil mid-fraction as cocoa butter replacers using differential scanning calorimetry and pulse nuclear magnetic resonance. Food Research International, 55: 103-109.

Kellens, M; Gibon, V; Hendrix, M and De Greyt, W (2007). Palm oil fractionation. European J. Lipid Science and Technology, 109: 336-349.

Kuntom, A; Siew, W L; Tan, Y I; Nor Aini, I and Ibrahim, N A (2005). Series P and Series F. $M P O B$ Test Methods. MPOB, Bangi. p. 55-58, 282-289, 366374.

Kushairi, A; Soh, K L; Azmani; Elina, H; Melina, O A; Zanal, B M N I; Razmah, G; Shamala Sundram, S and Ghulam, K A P (2018). Oil palm economic performance in Malaysia and R\&D progress in 2017. J. Oil Palm Res. Vol. 30(2): 163-195.

Lumor, S E; Prodriguez, P A M; Shewfelt, R L and Akoh, C C (2010). Physical and sensory attributes of a trans-free spread formulated with a blend containing a structured lipid, palm mid-fraction, and cottonseed oil. J. Amer. Oil Chem. Soc., 87(1): 6974.

Marangoni, A G (2005). The nature of fractility in fat crystal networks. Fat Crystal Networks. p. 413-440. 
Marangoni, A G and Lencki, R W (1998). Ternary phase behavior of milk fat fractions. J. Agricultural and Food Chemistry, 46: 3879-3884.

Minato, A; Ueno, S; Yano, J; Wang, Z; Seto, H; Amemiya, Y and Sato, K (1996). Synchrotron radiation $\mathrm{X}$-ray diffraction study on phase behavior of PPP-POP binary mixtures. J. Amer. Oil Chem. Soc., 73: 1567-1572.

Narine, S S and Marangoni, A G (1999). Relating structure of fat crystal networks to mechanical properties: A review. Food Research International, 32: 227-248.

PORAM Standard Specifications (2013). http: / / poram.org.my / p/wp-content / uploads / 2013/12/1.-PORAM-StandardSpecification.pdf, accessed on 29 November 2019.

Ramli, M R; Siew, W L and Cheah, K Y (2008). Properties of high-oleic palm oils derived by fractional crystallization. J. Food Science, 73: C140-C145.

Rashid, D N; Chiew, L C; Chong, S C and Omar, Z (2012). Crystallisation kinetics of palm stearin, palm kernel olein and their blends. LWT - Food Science and Technology, 46: 571-573.

Saadi, S; Ariffin, A A; Ghazali, H M; Miskandar, MS; Boo, H C and Abdulkarim, S M (2012). Application of differential scanning calorimetry (DSC), HPLC and $\mathrm{pNMR}$ for interpretation primary crystallisation caused by combined low and high melting TAGs. Food Chemistry, 132: 603-612.

Sivaruby, K; Enamul Hoque, M; Miskandar, M S and Spowage, A (2013). Investigating the effect of deforming temperature on the oil-binding capacity of palm oil based shortening. J. Food Engineering, 118: 90-99.

Sivaruby, K; Zaliha, O and Miskandar, M S (2018). Physical and chemical characteristics of palm oil-based solid fractions with more than $20 \%$ of tripalmitoyl-glycerol from Malaysian fractionation plants. J. Oil Palm Res. Vol. 30(3): 515-528.

Son, J M; Lee, K T; Akoh, C C; Kim, M R; Kim, M J and Lee, J H (2010). Optimisation of tripalmitin-rich fractionation from palm stearin by response surface methodology. J. Science of Food and Agriculture, 90: 1520-1526.

Sonwai, S; Kaphueakngam, P and Flood, A (2014). Blending of mango kernel fat and palm oil midfraction to obtain cocoa butter equivalent. J. Food Science and Technology, 51: 2357-2369.

Tan, C P and Che Man, Y B (2002). Differential scanning calorimetric analysis of palm oil, palm oil based products and coconut oil: Effects of scanning rate variation. Food Chemistry, 76: 89-102.

Toro-Vazquez, J; Dibildox-Alvarado, E; Herrera, V C and Charó-Alonso, M (2001). Triacylglyceride crystallization in vegetable oils: Application of models, measurements and limitations. Crystallization and Solidification Properties of Lipids (Widlak, N; Hartel, R and Suresh, N eds.). AOCS Press. p. 53-77.

Vereecken, J; De Graef, V; Smith, K W; Wouters, J and Dewettinck, K (2010). Effect of TAG composition on the crystallization behaviour of model fat blends with the same saturated fat content. Food Research International, 43: 2057-2067. 